\title{
A note on flasque sheaves
}

\section{J. M. Campbell}

We give an elementary and self-contained proof of the result that the sheaf $\tilde{M}$ determined by an injective module $M$ over a commutative noetherian ring is flasque.

Grothendieck shows in [1] (Corollary 2.7) that

the sheaf $\tilde{M}$ determined by an injective module $M$ over a commutative noetherion ring $A$ is flasque.

The proofs he gives use cohomology or relatively deep results on the structure of injective modules over commutative noetherian rings. The purpose of this note is to give an elementary and self-contained proof. We use the same notation and terminology as Macdonald [2]; in particular, the identity element of $A$ is denoted by 1 .

Let $U$ be an open subset of $\operatorname{Spec}(A)$. Then $U$ is the union of finitely many basic open subsets:

$$
U=\bigcup_{i=1}^{n} D\left(f_{i}\right)
$$

A section of $\tilde{M}$ over $U$ is then a family of elements

$$
s_{i}=\frac{m_{i}}{f_{i}} \in M_{f_{i}} \quad\left(m_{i} \in M, \quad f_{i} \in A ; i=1, \ldots, n\right)
$$

with the property that there exist integers $z_{i j} \geq 0$ such that

$$
\left(f_{i} f_{j}\right)^{i} i j\left(f_{i}^{k_{i}} m_{j}-f_{j}^{k}{ }_{m_{i}}\right)=0 \quad(i, j=1, \ldots, n) .
$$

Received 15 December 1969. Communicated by L.G. Kovács. 229 
Evidently, we may suppose that

$$
k_{i}=k, z_{i j}=\imath \quad(i, j=1, \ldots, n) \text {; }
$$

then (1) becomes

$$
\left(f_{i} f_{j}\right)^{2}\left(f_{i}{ }^{k} m_{j}-f_{j}{ }^{k} m_{i}\right)=0 \quad(i, j=1, \ldots, n) .
$$

To prove that $\tilde{M}$ is flasque we must show that there exists an element $m$ of $M$ and an integer $s \geq 0$ such that

$$
f_{i}^{s}\left(f_{i}^{k} m-m_{i}\right)=0 \quad(i=1, \ldots, n) \text {. }
$$

Consider, for $s \geq 0$, the system of equations

$$
f_{i}^{s+k} x=f_{i}^{s} m_{i} \quad(i=1, \ldots, n)
$$

in one unknown $x$. A solution $m$ in $M$ to (4) clearly satisfies (3).

If (4) were compatible, that is, if

$$
\sum_{i=1}^{n} a_{i} f_{i}^{s+k}=0 \text { implied } \sum_{i=1}^{n} a_{i} f_{i}^{s} m_{i}=0 \text { for all } a_{i} \in A \text {, }
$$

then the assignment

$$
f_{i}^{s+k} \mapsto f_{i}^{s} m_{i} \quad(i=1, \ldots, n)
$$

would define a homomorphism $\theta: L \rightarrow M$, where $L$ is the ideal in $A$ generated by $f_{i}^{s+k}(i=1, \ldots, n)$. But then, since $M$ is injective, $\theta$ could be continued to a homomorphism

$$
\bar{\theta}: A \rightarrow M,
$$

and the element $\bar{\theta}(1)$ would be a solution to (4).

It therefore suffices to prove that we can choose $s$ to make (4) a compatible system. We use induction on $n$, and suppose initially that $n=1$.

For $s \geq 0$, let $J_{s}$ be the ideal in $A$ consisting of all elements a such that 


$$
a f_{1}^{s} m_{1}=0 \text {. }
$$

Then $J_{s} \subseteq J_{s+1}$, and since $A$ is noetherian, we may choose $s$ so that

$$
J_{q}=J_{s} \text { for all } q \geq s
$$

If $a f_{1}{ }^{s+k}=0$ then $a \in J_{s+k}=J_{s}$, giving $a f_{1} s_{m_{1}}=0$. Thus (4) is compatible for this choice of $s$.

Now suppose that $n>1$. By induction, we may choose $s \geq 0$ so that the system

$$
f_{i}^{s+k} x=f_{i}^{s} m_{i} \quad(i=2, \ldots, n)
$$

is compatible. Then $M$ contains an element $m_{0}$ such that

$$
f_{i}^{s+k_{m_{0}}}=f_{i}^{s} m_{i} \quad(i=2, \ldots, n) \text {. }
$$

Let $K_{s}$ be the ideal in $A$ consisting of all elements $a$ such that

$$
a f_{1}^{s}\left(m_{1}-f_{1}{ }^{k} m_{0}\right)=0 \text {. }
$$

Then $K_{s} \subseteq K_{s+1}$, and we may evidently choose $s$ so that (5) remains valid, $K_{q}=K_{s}$ for all $q \geq s$, and $s \geq \imath$.

We claim that (4) is a compatible system for this choice of $s$. In fact, let

$$
\sum_{i=1}^{n} a_{i} f_{i}^{s+k}=0
$$

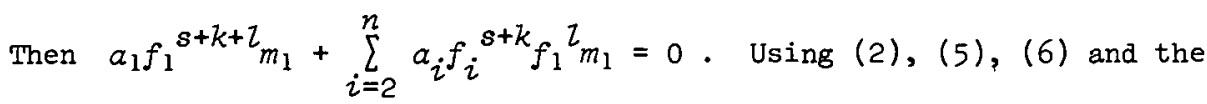
fact that $s \geq \imath$, we obtain

$$
a_{1} f_{1}^{s+k+2}\left(m_{1}-f_{1} m_{0}\right)=0
$$

But then $a_{1} \in K_{s+k+2}=K_{s}$, so that 


$$
a_{1} f_{1}^{s}\left(m_{1}-f_{1} k_{0}\right)=0
$$

Using (5) and (6), we obtain

$$
\sum_{i=1}^{n} a_{i} f_{i}^{s} m_{i}=0
$$

as required for compatibility. This completes the proof.

\section{References}

[1] A. Grothendieck, Local cohomology (Lecture Notes in Mathematics 41, Springer-Verlag, Berlin, Heidelberg, New York, 1967).

[2] I.G. Macdonald, Algebraic geometry (W.A. Benjamin, New York, Amsterdam, 1968).

Canberra College of Advanced Education,

Canberra, ACT. 